\title{
Voltage control method for distribution networks based on active load transfer
}

\author{
Baoxuan Ye ${ }^{\mathrm{a}}$, Yuwu Wu ${ }^{\mathrm{a}}$, Bingzhang Tong ${ }^{\mathrm{a}}$, Kangjian Wang ${ }^{\mathrm{a}}$, Luqing Wang ${ }^{\mathrm{a}}$, \\ Shijian Zhang ${ }^{\mathrm{b}}$ \\ ${ }^{a}$ Hainan power grid Co., Ltd, Wenchang 571300, Hainan, China \\ ${ }^{b}$ BENLIU Electrical Technology Co., Ltd, Guangzhou 510640, Guangdong, China
}

\begin{abstract}
The existing reactive power and voltage control methods are temporarily unable to meet the normal voltage regulation needs of distribution network. Aiming at the status quo, a new voltage control method based on active load transfer, is proposed in this paper, according to the typical connection structure and voltage control analysis of distribution network. This method takes the highest nodal voltage qualification rate of the line in a time period as the objective function, establishes voltage control model of active load transfer, and solves the model by extending the calculation, so as to maintain the line voltage within the qualified range. Taking a real feeder as an example, the traditional voltage control method and the method presented in this paper are simulated and compared, which proves validity of the proposed method, which performs excellently in reducing power loss and improving voltage quality.
\end{abstract}

Keywords: active distribution network; voltage control; active load transfer

\section{Introduction}

The distribution networks with large-scale, high-permeability distributed generation (DG) has become a trend in the power industry [1], and has completely changed the structure of distribution network, which was a traditional single-source radial structure and now is a complex structure rich of power supplies. Therefore, the effective method of voltage control for distribution network has become one of the current research hotspots [2-4], which has a great significance in loss reduction and voltage-quality improvement.

Many scholars have studied the voltage control of distribution network [5-10]. The article [6] proved that the point the DG accesses to the grid is most likely to beyond voltage limitation and proposed a local voltage control strategy based on both active and reactive power regulation. The article [7] proposed a voltage control strategy suitable for angular cascade STATCOM in large photovoltaic power station, which can reduce voltage fluctuations at the common connection point. The article [8] proposed an areal voltage control method, which cooperates with static synchronous compensator and DG. Most of the current researches focus on the voltage control at the device level, and generally set the DG controllable. However, in the actual operation, the controllability of the DG is relatively weak, and some regions are lack of device-level voltage control resources. When the high-permeability DG is accessed, the above control method cannot obtain ideal voltage control benefit. In this paper, aiming at the above problem, a voltage control method based on active load transfer is proposed, according to which, firstly establish the voltage control model of active load transfer and then deduces it with mathematical extension.

\section{Typical Wiring Structure of Distribution Network}

The typical wiring structure can be divided into two types depending on whether or not they have load

\footnotetext{
* Manuscript received March 15, 2018; revised August 19, 2018.

Corresponding author: Tel.: +86-18826076283; E-mail address: $1870453691 @ q q . c o m$.

doi: $10.12720 /$ sgce.8.5.592-596
} 
transfer capability. The first type is basic wiring, also called single radiation wiring, which has no interconnection switch and load transfer capability. The second type, connection wiring, has the capability because there are one or more sectional switches and interconnection switches on the line. The connection wiring includes "n-1" single ring network, $\mathrm{N}$ power supplies with an emergency supply, double ring network, multi-sectioned and multi-linked wiring, petal type wiring, last two of which are rarely used in China's distribution network. However, at this stage, the load transfer is still considered to strengthen the reliability of distribution network, such as fault isolation and power supply restoration. There is little research about its impact on the operation and control of distribution networks.

\section{Analysis of Voltage Control for Distribution Networks}

In the traditional distribution network, power is transmitted in one direction, and the voltage drops from the head of the feeder to the end. In an active distribution network with DG, due to the influence of the power that DG outputs, the voltage drop may be from the end of the feeder to the head, and the voltage where DG accesses to grid may be the highest, which may even be beyond the voltage limits. In particular, seasonal long-term excessive voltage may occur in the feeder rich of small hydropower.

Most of traditional methods still take more account from the device level for the voltage control of the distribution network, such as using capacitors, reactors, SVG, energy storage and so on for voltage regulation. In the distribution network with small-capacity DG, reactive power compensation can meet the requirement of voltage control. However, the reactive power compensation device is not configured for some nodes of distribution transformer limited by configuration principle. Additionally, the current guide rules of the devices mainly focus on capacitive rather than inductive reactive power compensation. Therefore, in distribution networks with limited voltage regulation equipment but high-permeability DG, especially in the grid that has long-term overvoltage caused by the connection of small-scale hydropower, voltage control strategy is not effective enough, although the equipment of voltage regulation is used up.

\section{Voltage Control of Active Load Transfer}

Aiming at the shortcomings in the current wiring structure and voltage control of distribution networks, this paper proposes a voltage control method based on active load transfer, the control model, mathematical expansion and implementation flow of which are as follow.

\subsection{Voltage control model}

The aim of voltage control based on active load transfer is to solve the problem of seasonal distribution network overvoltage that the current voltage regulation methods cannot solve. Therefore, the objective function is the highest qualified rate of nodal voltage within a certain time period, and the model is:

$$
\min f=1-\frac{\sum_{i=1}^{t} k_{i}}{t \bullet k_{\Sigma}}
$$

In the formula, $k_{i}$ : The number of load nodes whose voltage are qualified at a certain time section; $k_{\Sigma}$ : The total of load nodes on the feeder; $t$ : The total of time sections during a continuous time period.

The equality constraint is power flow constraint, and the inequality constraints include line overload, transformer overload, the length of feeder, etc. And it is shown as follow: $\left\{\begin{array}{l}h(x)=0 \\ S_{l} \leqslant S_{l \max } \\ S_{t} \leqslant S_{t \max } \\ L \leqslant L_{\max }\end{array}\right.$

In the formula, $h$ : The power flow constraint; $S_{l}$ and $S_{l \max }$ : The calculated value and the maximum permissible value of the line power; $S_{t}$ and $S_{t \max }$ : The calculated value and the maximum permissible 
value of the power out of a transformer; $L$ and $L_{\max }$ : The total and the maximum permissible value of the length of the line.

\subsection{Mathematical expansion}

The above voltage control model can use optimization algorithm to solve, but it requires the model of line to be relatively accurate and concrete, which makes the solution difficult. But it is noteworthy that the above model is well-targeted and has a single target object, the overvoltage feeders with DG, which has a few solution elements. In the distribution network, the wirings such as "2-1", "3-1", and "N-sectioned and $\mathrm{n}$-linked $(\mathrm{N} \leqslant 6, \mathrm{n} \leqslant 3)$ " are generally used, and it is not a huge set of possible wirings after load transfer, at less, it pertains to the same order of magnitude as the target object. So, it is reasonable to expand the formula of active load transfer model and select scheme with constraint formula to obtain the results.

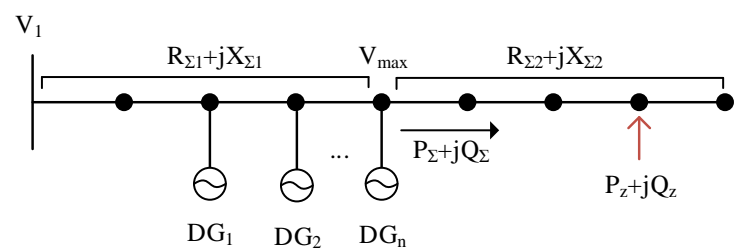

Fig. 1 Equivalent model of active load transfer for distribution Network with multi-DG

Taking the distribution network with multi-DG as shown in Fig. 1 as an example, now make the objectives of the above control model further limited. After the load transfer, the operating voltage is controlled within a qualified range. The load capacity transferred after expansion is as shown in Eq. (3).

$$
\left\{\begin{array}{l}
\frac{\left(V_{\max }-10.7\right) * V_{1}}{R_{\Sigma 1}}<P_{Z 1}<\frac{\left(V_{\max }-V_{x}\right) * V_{1}}{R_{\Sigma 1}} \\
V_{x}-\frac{P_{\Sigma} R_{\Sigma 2}+Q_{\Sigma} X_{\Sigma 2}}{V_{x}}>9.3, \quad L_{3}<L_{1} \\
V_{x}-\frac{L_{3}+L_{4}-L_{1}}{L_{2}} \times \frac{P_{\Sigma} R_{\Sigma 2}+Q_{\Sigma} X_{\Sigma 2}}{V_{x}}>9.3, \quad L_{3} \geqslant L_{1}
\end{array}\right.
$$

In the formula, $V_{\max }$ : The highest of nodal voltage; $V_{1}$ : Nodal voltage of the head of the feeder; $R_{\Sigma 1}$ : The resistance from the head of the feeder to the node with highest voltage; $R_{\Sigma 2}$ and $X_{\Sigma 2}$ : The resistance and reactance from the node with highest voltage to the end of the feeder; $P_{\Sigma}$ and $Q_{\Sigma}$ : The total active power and reactive power which transferred from the node with highest voltage to the end; $P_{Z 1}$ : The active power of load which is transferred; $L_{1}$ : The distance from the head of the feeder to the node with highest voltage; $L_{2}$ : The distance from the node with highest voltage to the end of the feeder; $L_{3}$ : The distance from the node which load is transferred to the head of the feeder; $L_{4}$ : length of the line corresponding to load transfer amount. $V_{x}$ : The reference value of voltage, determined by Eq. (4), in which, $L=L_{1} / L_{2} ; \bar{V}$ and $\underline{V}$ are the upper and the lower limit of nodal voltage

$$
V_{x}=\left\{\begin{array}{lr}
V_{\max }+\left(\frac{1}{3}-L\right)\left(\bar{V}-V_{\max }\right), & L \leqslant \frac{1}{3} \\
V_{\max }+\left(\frac{1}{2}-L\right)(\bar{V}-\underline{V}), & \frac{1}{3}<L<\frac{2}{3} \\
V_{\max }+\left(L-\frac{1}{3}\right)\left(\underline{V}-V_{\max }\right), & L \geqslant \frac{2}{3}
\end{array}\right.
$$


To avoid interconnection switches action frequently, this paper expands it to a confidence interval of load capacity transferred at continuous time period, defined as $P_{\mathrm{CCON}}$, which is calculated as follow:

1)Select the maximum and minimum of nodal voltage at a single time section, defined as $V_{\max }$ and $V_{\min }$ respectively, with which, the first and second confidence interval of load capacity transferred, defined as $P_{Z 1}$ and $P_{Z 2}$ respectively can be calculated and obtained.

2)Obtain the confidence interval of load capacity transferred $P_{C C O N}=P_{Z 1} \cap P_{Z 2}$

The final plan of load transfer can be determined after subsequent examination.

\subsection{Implementation flow}

According to the above formula expansion about the model of active load transfer, the confidence interval of load capacity transferred can be obtained, from which, it is possible to filtered transfer lines and achieve voltage control based on active load transfer. The specific steps are as follow:

- Obtain basic line data, historical operation data, and information about the point DG access to the grid;

- Calculate the confidence interval of load capacity transferred PCCON;

- filter the elements in the set $\mathrm{A}$, which is of interconnection lines with load transfer,

- Output the elements in the set $\mathrm{A}$ that meet the inequality constraints to the set B, whose elements are lines that can achieve load transfer.

- Select an element in set B for voltage control of load transfer.

\section{Simulation}

A group of $10 \mathrm{kV}$ ring network lines in the southern region is selected as the example. Now verify the effect of the active load transfer method the paper proposed in reducing power loss and improving line voltage through comparative analysis. The wiring structure of this ring group is shown in Fig. 2.

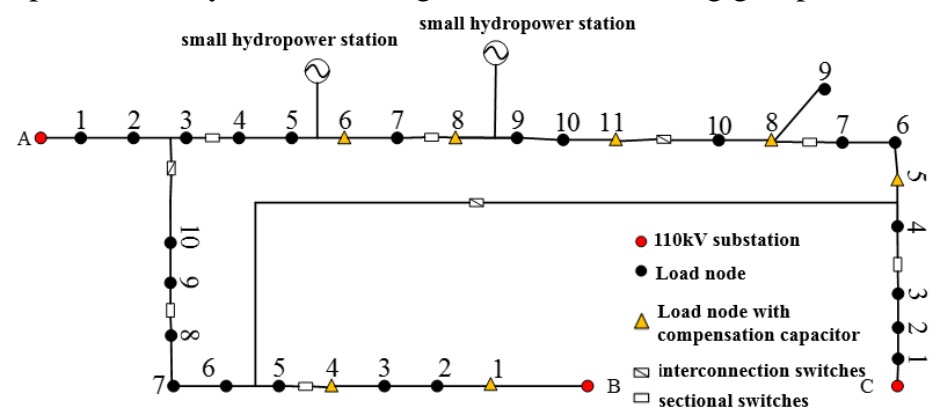

Fig. 2 Wiring of the networks for simulation

The ring networks, contains 31 nodes of distribution transformer, is a township line and the total capacity of transformers is $12250 \mathrm{kVA}$. There are two small hydropower stations on line A, whose capacity their access are 5.5 MW and $3 \mathrm{MW}$ respectively. To strengthen the contrast effect, the line load level, the position where DG access and their capacity, the configuration capacitor are exactly the same din two simulations with different methods. One of the voltage control methods is traditional and the other is based on active load transfer. The simulation results are shown in Table 1.

Table 1. Comparison of the results of the two control methods

\begin{tabular}{|c|c|c|c|c|}
\hline Method & $\begin{array}{c}\text { Total loss of active } \\
\text { power/kW }\end{array}$ & Highest nodal voltage $/ \mathrm{kV}$ & Lowest nodal voltage $/ \mathrm{kV}$ & voltage qualification rate $/ \%$ \\
\hline traditional & 343 & 10.83 & 10.01 & 63.3 \\
\hline $\begin{array}{c}\text { proposed in the } \\
\text { paper }\end{array}$ & 235 & 10.52 & 9.98 & 100 \\
\hline
\end{tabular}


As is shown in the above simulation results, the method based on active load transfer the paper proposed for distribution network voltage control is superior to traditional voltage control method in terms of energy saving, loss reduction and voltage quality improvement.

In terms of network loss, the control method proposed in this paper is reduced by $31.5 \%$ compared to the traditional method; in terms of voltage quality, the control method proposed in this paper can effectively maintain the voltage of the feeder within the qualified range and increase the voltage qualification rate to $100 \%$.

\section{Conclusion}

Aiming at the current status of wiring structure and voltage control in distribution network in China, this paper proposes a voltage control method based on active load transfer. Through the simulation and calculation of the traditional method and the proposed method, the results show that under the same simulation environment, the proposed method has a remarkable effect on power quality control, and has a better operating economic benefit. This method does not rely on accurate modeling of distribution networks and complex optimization algorithms. It is easy to operate and does not need to increase investment in voltage control resources of distribution networks. It is economical and practical, especially for seasonal long-term overvoltage lines with small hydropower.

\section{References}

[1] Eltantawy AAB, Salama MMA. Management scheme for increasing the connectivity of small-scale renewable DG. IEEE Transactions on Sustainable Energy, 2014; 5(4): 1108-1115.

[2] Wang XX, Xu T, Wang CS, et al. Distributed voltage control in active distribution networks utilizing multiple agent system. Proceedings of the CSEE, 2016; 36(11): 2918-2926.

[3] Chen F, Liu D, Chen YH. Hierarchically distribution voltage control strategy for active distribution network. Automation of Electric Power Systems, 2015; 39(09): 61-67.

[4] Cai Y, Lin J, Song YH, et al. Voltage control strategy in active distribution network based on model predictive control. Transactions of China Electrotechnical Society, 2015; 30(23): 42-49.

[5] Sansawatt T, O' Onnell J, Ochoa LF, et al. Decentralised voltage control for active distribution networks. In: Proc. of the 44th International Universities Power Engineering Conference(UPEC), September 1-4, 2009, Glasgow, UK:5p.

[6] Wei HK, Liu J, Gao H. Local voltage control of distributed generations. Electric Power Automation Equipment,2016; 36(9): $40-45$.

[7] Xie N, Chen XK, Xu XG, etc. Voltage control strategy for delta-connected cascaded TATCOM oflarge-scale photovoltaic power station. Electric Power Automation Equipment; 2017(3): 61-66.

[8] Zhang WY, Li YL, Sun GY, etc. Zonal-voltage control for active distribution network ased on distributed static synchronous compensator. Proceedings of the CSEE, 2015; 35(7): 1644-1656.

[9] Bonfiulio A, Briunone M, Delfino F. Optimal control and operation of grid-connected photovoltaic production units for voltage support in medium-voltage networks. IEEE Trans on Sustainable Energy. 2014; 5(1): 254-263.

[10] Leisse I, Samuelsson O, Svensson, J. Coordinated voltage control in distribution systems with DG-control algorithm and case study. CIRED Workshop, May 29-30, 2012, Lisbon, Portugal: 4. 